\title{
Corpus Cavernosum Atypical Metastasis of Renal Cell Carcinoma: Presurgical Therapy and Complete Surgical Resection. Case Report and Review of the Literature
}

\author{
Saccà $A^{1 \#^{*}}$, Roscigno $M^{1 \#}$, Nicolai $M^{1}$, Manica $M^{1}$, La Croce $G^{1}$, Bettini $A^{2}$, Chirco $A^{2}$, Bonomi $L^{2}$ and Da \\ Pozzo L ${ }^{2}$
}

${ }^{1}$ Department of Urology, ASST Papa Giovanni XXIII, Bergamo, Italy

${ }^{2}$ Department of Oncology, ASST Papa Giovanni XXIII, Bergamo, Italy

${ }^{\#}$ Equally contributed.

*Corresponding author: Antonino Saccà, Department of Urology, ASST Papa Giovanni XXIII, Piazza OMS1, 24127 Bergamo, Italy, Tel: +393387720315, Fax: +390352674888, E-mail: dr.antoninosacca@gmail.com

\begin{abstract}
Renal-Cell Carcinoma (RCC) accounts for $2 \%$ of all cancers. Metastases are present in up to $30 \%$ of patients at diagnosis or appeared during follow up [1]. Atypical RCC metastases are sites, other than chest, liver, bone, adrenal, brain, and nodes, and their presentation is very rare.

We describe the case of a 71-years-old male, who was submitted to a left laparoscopic radical nephrectomy for Clear Renal Cell Carcinoma (CRCC). After a 14-year follow-up, MRI scan showed a $5-\mathrm{cm}$ solid lesion of the perineum, that originates from the right corpus cavernosum. A percutaneous trans-perineal biopsy revealed an atypical metastasis of CRCC. To reduce surgical risk during the exeresis of the mass, the patient received 4 cycles of pazopanib as presurgical treatment, with a shrinking of the lesion at follow-up MRI scan. He underwent a complete resection of the residual mass. Histopathology confirmed the diagnosis of atypical corpus cavernosum metastasis of CRCC. Presurgical therapy with Target Molecular Therapy (TMT) is feasible and might have several potential advantages for patients in case of complex metastasectomy.
\end{abstract}

\section{Keywords}

Corpus cavernosum, Presurgical therapy, Metastatic renal cell carcinoma, Multimodal therapy, Metastasectomy

\section{Introduction}

Up to $30 \%$ of RCC patients are metastatic at diagnosis or metastases may be discovered during follow-up [1-3]. The major sites of metastasis include lungs, brain, bones, liver, adrenal glands and nodes, while atypical locations are very much rare. Antonelli, et al. described, in their long time single-center experience, the main atypical sites of RCC metastasis, that are skin, thyroid, muscles, pancreas, testicle [4]. The treatment of atypical metastasis has not been yet clearly defined: European Association of Urology (EAU) guidelines do not currently take into consideration the treatment of atypical RCC metastases because they are relegated to limited experience and case reports [5]. The role of surgery has to be considered useful due to the lack of other efficacious medical therapies, and for the limited ability of new TMT to produce complete regression and for their related multiple side effects $[2,5,6]$.

\section{Case Report}

We present the case of a 71-years-old Caucasian male, with a past medical history of hypertension, Benign Prostatic Hyperplasia (BPH) and previous inguinal hernia repair. In November 2002, he underwent a laparoscopic left radical nephrectomy for a 10-cm CRCC of the left kidney with perirenal fatty tissue involvement and thrombosis of the left renal vein (pT3b NO). Patient was followed up regularly on the basis of the EAU guidelines on renal cancer. After a 14-year follow-up he presented a painless, irregular, solid mass of the perineum. MRI scan of the pelvis and perineum revealed a disho- 


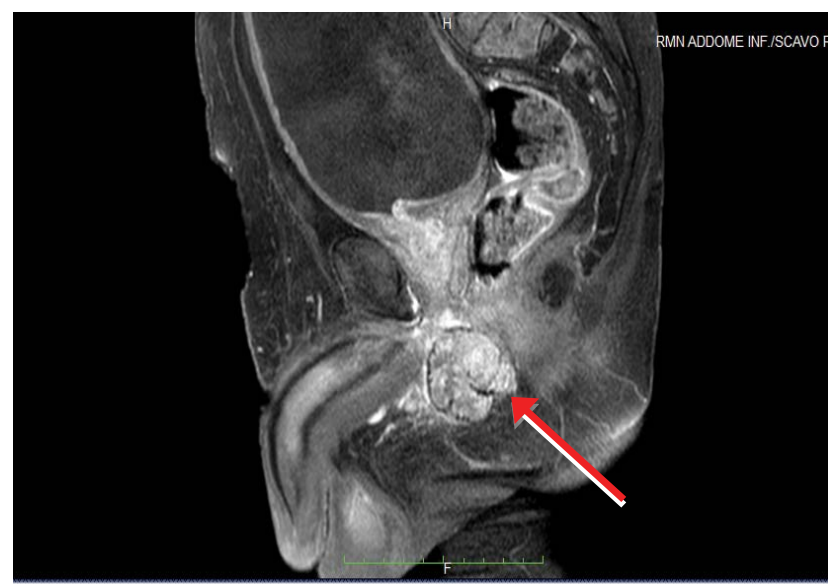

Figure 1a: MRI scan revealed a solid expansive lesion from the crus of right corpus cavernosum (red arrow); the mass reached the limit of the membranous urethra.

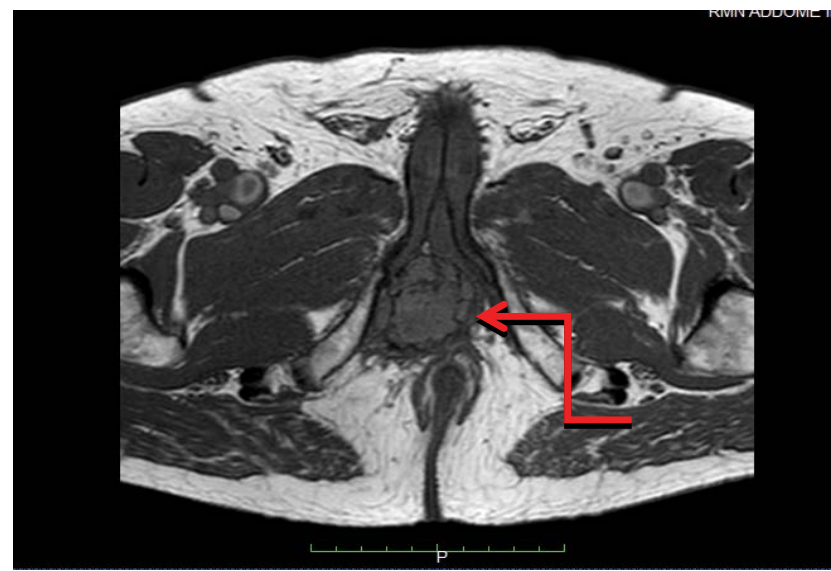

Figure 1b: MRI scan revealed a solid expansive lesion from the crus of right corpus cavernosum (red arrow); the mass was placed posteriorly to the base of the penis and strictly related to the internal profile of the right ischio-pubic branch.

mogeneous solid expansive lesion with polylobate profiles of $52 \times 58 \mathrm{~mm}$, that originated from the crus of right corpus cavernosum: The mass was placed posteriorly to the base of the penis and strictly related to the internal profile of the right ischiopubic branch with partial compression and dislocation to the left of the anal canal and of the corpus spongiosum of the urethra (Figure 1a and Figure $1 \mathrm{~b})$. Moreover, the mass reached the limit of the membranous urethra. A trans-perineal ultrasound guided biopsy of the lesion was performed, and the pathological result showed a metastasis of CRCC. A multi-disciplinary meeting was scheduled. The patients presented favorable prognosis according to Heng and Memorial Sloan-Kettering Cancer Center (MSKCC) criteria and would have benefited from surgical resection of this single metastasis. In order to reduce surgical risk during the exeresis of the mass, the patient was treated with 4 cycles of pazopanib, as presurgical modality. Adverse effects were $\mathrm{G} 1$ increase in blood pression and grade 2 diarrhea symptoms. In April 2017, a follow-up-scheduled MRI scan demonstrated volumetric a $30 \%$ reduction of the perineal lesion (Figure $2 \mathrm{a}$ and Figure $2 \mathrm{~b}$ ). Thus, in

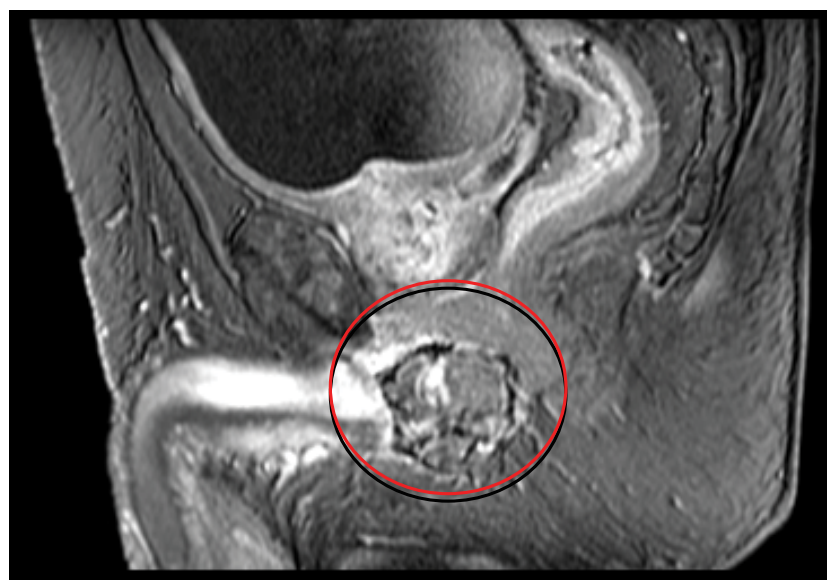

Figure 2a: Sagittal MRI scan demonstrated a $30 \%$ volumetric reduction of the perineal mass (red circle).

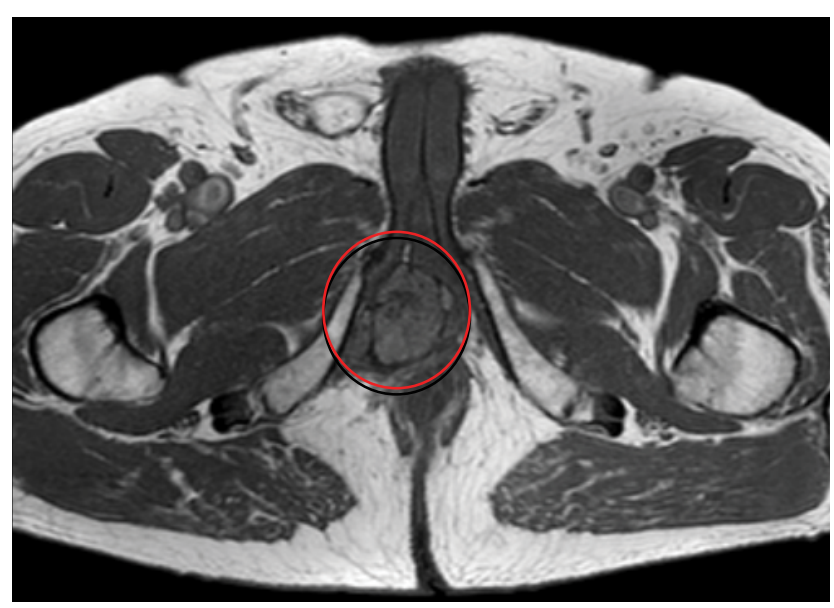

Figure 2b: Axial MRI scan demonstrated a $30 \%$ volumetric reduction of the perineal mass (red circle).

June 2017, this patient underwent metastasectomy of the perineal mass by a median perineal access. The mass was smoothly dissected from the corpus cavernosum and the corpus spongiosum of the urethra (Figure 3a, Figure $3 \mathrm{~b}$ and Figure $3 \mathrm{c})$. The final pathological report revealed metastatic carcinoma from the right corpus cavernosum, compatible with RCC dissemination, and with negative surgical margins. According to complete resection, no TMT was proposed after surgery. At 12-month follow-up, the patient is in general good health status, and total body CT scan showed no recurrences.

\section{Discussion}

Renal cell carcinoma is diagnosed metastatic up to $30 \%$ of cases or could develop metastases during follow-up, even with late onset [1].

Synchronous or metachronous RCC metastases typically occur to the brain, lungs, bones, liver, adrenals; atypical location appears to be rare and may involve numerous distant sites [4]. Corpus carvernosum is intended to be an atypical site and is usually synchronous with the primary renal lesion [7], while if it is presented as metachronous, the onset is temporally close to the renal cancer diagnosis $[8,9]$. 


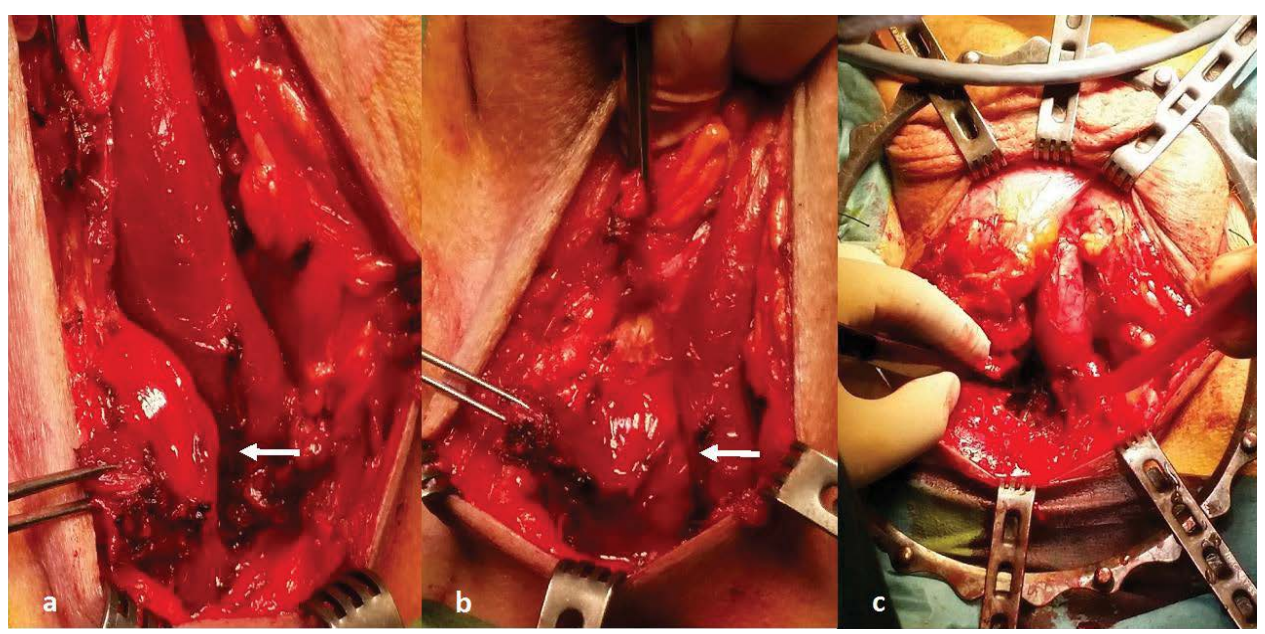

Figure 3: $a, b)$ The mass was dissected from the corpus cavernosum and the corpus spongiosum (white arrow points the mass and its relations to corpus cavernosum (a), and corpus spongiosus (b); c) Surgical field after complete resection of the metastasis.

We present an extremely late onset of CRCC metastasis to corpus cavernosum. Fourteen years from the diagnosis represents a delayed onset despite initial renal histological examination presenting an aggressive disease. A longer follow-up has to be considered in front of initial poor prognosis renal cancers.

The timing and role of metastasectomy in the TMT era remain an unanswered question: EAU guidelines consider the surgery of renal metastasis in patients with a favourable risk profile in whom complete resection is achievable [4]. As suggested by Kavolius, et al. [2], an interval from RCC diagnosis to occurrence of metastases $>1$ year, a unique metastatic site, and age $<60$ year have been identified as favorable survival predictors following RCC metastases resection. Recently, Ball, et al. [10] confirmed the usefulness of systemic therapy before metastasectomy in solitary or oligometastatic patients and when complete resection is feasible.

A CRCC metastasis to the corpus cavernosum is a very rare situation, and we aim to stress the role of a multidisciplinary approach, that is the discussion of every single clinical case among different specialists, i.e. urologists, uro-oncologists and radiotherapists, in order to choose the best therapeutic option tailored to each patient. According to preoperative staging images, the mass was strictly adjacent to the right corpus cavernosum, the corpus spongiosum of the urethra, and reached the limit of the membranous urethra. For this reason, we decided for a preoperative oncological treatment to allow a following complete surgical resection, while reducing the risk of surgical complications.

Patient is free from recurrence after 12 months. Longer follow up is needed in front of initial aggressive disease. However, the multimodality approach represents the cornerstone of a successful treatment that allows reducing side effect and surgical risks.

\section{References}

1. Rini BI, Campbell SC, Escudier B (2009) Renal cell carcinoma. The Lancet 373: 1119-1132.

2. Kavolius JP, Mastorakos DP, Pavlovich C, Russo P, Burt $\mathrm{ME}$, et al. (1998) Resection of metastatic renal cell carcinoma. J Clin Oncol 16: 2261-2266.

3. Nguyen MM, Gill IS (2009) Effect of renal cancer size on the prevalence of metastasis at diagnosis and mortality. $J$ Urol 181: 1020-1027.

4. Antonelli A, Arrighi N, Corti S, Legramanti S, Zanotelli T, et al. (2012) Surgical treatment of atypical metastasis from renal cell carcinoma (RCC). BJU International 110: E559-E563.

5. Ljungberg $B$, Bensalah $\mathrm{K}$, Canfield $\mathrm{S}$, Dabestani $\mathrm{S}$, Hofmann F, et al. (2015) EAU guidelines on renal cell carcinoma: 2014 update. Eur Urol 67: 913-924.

6. Karam JA, Rini BI, Varella L, Garcia JA, Dreicer R, et al. (2011) Metastasectomy after targeted therapy in patients with advanced renal cell carcinoma. J Urol 185: 439-444.

7. Nezu FM, Dhir R, Logan TF, Lavelle J, Becich MJ, et al. (1998) Malignant priapism as the initial clinical manifestation of metastatic renal cell carcinoma with invasion of both corpora cavernosum and spongiosum. Int J Impot Res 10: 101.

8. Romero Selas E, Lamas Meilán C, Barbagelata López A, Ponce Díaz-Reixa JL, Fernández Rosado E, et al. (2006) Metastasis of a renal cell carcinoma in the corpora cavernosum of the penis. Case report and bibliographic review. Arch Esp Urol 59: 530-532.

9. Patard JJ, Pignot G, Escudier B, Eisen T, Bex A, et al. (2011) ICUD-EAU International Consultation of Kidney Cancer 2010: Treatment of metastatic disease. Eur Urol 60: 684-690.

10. Ball MW (2017) Surgical management of metastatic renal cell carcinoma. Discov Med 23: 379-387.
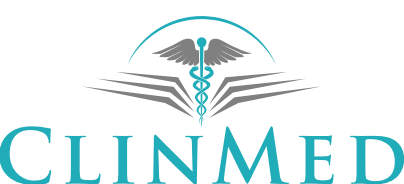

INTERNATIONAL LIBRARY 